\title{
FREQUENCY OF PROTEUS MIRABILIS INFECTION IN RHEUMATOID ARTHRITIS
}

\author{
Muhammad Hasan Saleem, Shazia Nisar*, Muhammad Zulqurnain Saleem, Husnain Saleem**, Shoaib Mukhtar***, \\ Muhammad Ali Anjum \\ Combined Military Hospital Kharian/National University of Medical Science (NUMS) Pakistan, *Pak Emirates Military Hospital/National University of Medical \\ Sciences (NUMS) Rawalpindi Pakistan, ${ }^{* *}$ Combined Military Hospital Peshawar/National University of Medical Science (NUMS) Pakistan, ${ }^{* * *}$ Armed Forces \\ Institute of Rehabilitation Medicine / National University of Medical Science (NUMS) Rawalpindi Pakistan,
}

\section{ABSTRACT}

Objective: To assess the frequency of proteus mirabilis infections in patients of rheumatoid arthritis.

Study Design: Cross-sectional study.

Place and Duration of Study: Combined Military Hospital, Rawalpindi Pakistan, from Jul 2018 to Jan 2019.

Methodology: A total of 100 cases of rheumatoid arthritis with urinary tract infection were enrolled. Freshly voided urine samples were obtained and sent to the pathology lab. The serotypes of proteus mirabilis were evaluated by enzyme-linked immunosorbent assay (ELISA) and immunoblotting and presence of proteus mirabilis was noted.

Results: A total of 100 cases of rheumatoid arthritis with urinary tract infection were recruited. The mean age of patients was $61.22 \pm 10.18$ years. There were total 67 (67\%) females and $33(33 \%)$ were males. About 79 (79\%) patients had sedentary lifestyle while 21 (21\%) had active lifestyle. There were $41(41 \%)$ patients had positive urine culture with proteus mirabilis while $59(59 \%)$ had other organism involved in urine infection.

Conclusion: The high frequency of proteus mirabilis infection has been detected and the frequency was of females with complaint of urinary tract infection was high.

Keywords: Proteus mirabilis, Rheumatoid arthritis, Rheumatoid factors, Urinary tract infection.

This is an Open Access article distributed under the terms of the Creative Commons Attribution License (https://creativecommons.org/licenses/by-nc/4.0/), which permits unrestricted use, distribution, and reproduction in any medium, provided the original work is properly cited.

\section{INTRODUCTION}

Microorganisms residing inside the human body develop a complex eco-system. The conjoint impact of microbes and immune system of the human body or host establish the beginning of several diseases like inflammatory bowel disease, obesity, pseudomembranous colitis, atopic diseases, type 1 diabetes or reactive arthritis ${ }^{1}$. New molecular diagnostic techniques and multi-center trials may support to understand the role of microbiota in health \& disease. Rheumatoid arthritis (RA) has multi-faceted cause and is under debate ${ }^{2,3}$.

Indications showed the significant impact of microbiomes residing in oral cavity, lungs, intestines and urinary tract on initiation of rheumatoid arthritis. Contacts between microbiomes and human immunity, is important in pathogenesis of rheumatoid arthritis ${ }^{4}$.

Proteus-mirabilis (P.mirabilis) got great fascination of micro-biologists for $>100$ years with its distinctive flooded diversity, dienes line construction and powerful urease activity. Transcriptome outlining during both; host infection \& swarming motility, coupled with availability of comprehensive genome sequence for $P$. mirabilis, has exposed the existence of interbacterial struggle and killing via type VI secretion system, and

Correspondence: Dr Muhammad Hasan Saleem, 595-G, Shah Rukn-eAlam Colony, Multan Pakistan

Received: 29 Dec 2019; revised received: 25 Aug 2020; accepted: 30 Sep 2020 joint regulation of bond and motility, along with close links between metabolism, swarming and virulence ${ }^{5}$. Patients with RA are reported to have in their sera raised levels of antibody specific to P.mirabilis. Infection of P.mirabilis bacteriuria in RA patients was $52 \%{ }^{6}$.

In recent methods for the management of RA normally do not consider the early stages of development of the disease and as a substitute only symptoms have been treated. At the same time as, this method might help to relieve the pain considerably as well as the discomfort related to the RA. But this method could not avert the progression of the disease and further destruction of joints tissues happens as a sequel. Additionally, several number of severe adverse effects occur due to prolonged intake of the current available treatment methods. One of the well improved approach might be applied to get rid of the source of the RA, thus evading the events of later phase. Still in routine, it has not been probable because of the lack of understanding of the causes of the RA 7,8 .

Rationale of this study was to assess the frequency of P.mirabilis infections in patients of RA belong to local community. P.mirabilis is the major bacterium involved in RA. But there was a lack of evidence regarding the extent of P.mirabilis infections in local community. So we conducted this study to find the commonness of the P. mirabilis in patients of RA. 


\section{METHODOLOGY}

This cross-sectional study was conducted at department of Medicine, Combined Military Hospital, Rawalpindi, from July 2018 to January 2019 after taking permission from Institutional Ethics Review Board (IERB no. 24855291). Cases aged 35-85 years, either gender of RA (positive RA factor) presenting with complaint of urinary tract infection (on urine examination) were included in the study by using non-probability, consecutive sampling technique. Patients having renal failure or disease (creatinine $>1.5 \mathrm{mg} / \mathrm{dl}$ ), renal carcinoma, patients with recurrent infection within 3 months of treatment were excluded. Sample size of 100 cases were calculated with 95\% confidence level, 10\% absolute precision and percentage of $P$. mirabilis i.e. $52 \%$ in patients with RA presenting with complaint of urinary tract infection. Informed consent and demographics were noted. Freshly voided urine samples were obtained by using a sterile container under aseptic measures. All samples were sent to the laboratory of the hospital to assess bacterium present in P.mirabilis. The entire drawn blood samples were centrifuged and stored in ice room. The serotypes of P.mirabilis were evaluated by enzyme-linked immunosorbent assay (ELISA) and immunoblotting and presence or absence of P.mirabilis was confirmed.

Analysis plan: All the information was entered electronically to Ms Excel Sheets and analyzed by using SPSS-21. Descriptive statistics was calculated i.e. mean \pm SD for quantitative variables and frequency \& percentage for qualitative variables.

\section{RESULTS}

The mean age of the patients was $61.22 \pm 10.18$ years. There was a total of $67(67 \%)$ females and 33 (33\%) were males. About 79 (79\%) patients had sedentary lifestyle while $21(21 \%)$ had active lifestyle. The duration of RA diagnosis was $21.36 \pm 8.91$ years. The mean duration of urinary tract infection symptoms was $25.67 \pm 9.14$ days (table-I).

The most common symptom of urinary tract infection was cloudy urine 89 , followed by burning sensation during urination (82\%), lower abdominal pain $77 \%$, persistent urge to urinate in $71 \%$, pink or cola color urine in $69 \%$ while 59 had passing frequent urine (table-II).

There were 41 patients had positive urine culture with P.mirabilis while 59 had other organism involved in urine infection (figure).
In all the urine samples, other than P. mirabilis, E. coli was detected in 21 cases, acinobacter species in 14 cases, klebsiella species in 10 cases, streptococcus species in 8 cases, enterococcus spp. In 5 cases while pseudomonas aeruginosa in 1 cases (table-III).

Table-I: Summary of demographic and diagnostic characteristics $(n=100)$.

\begin{tabular}{|c|c|c|}
\hline Parameters & & Values \\
\hline Age $($ mean \pm SD) & \multicolumn{2}{|c|}{$61.22 \pm 10.18$ years } \\
\hline \multicolumn{3}{|l|}{ Gender } \\
\hline Male & & 33 \\
\hline Female & & 67 \\
\hline \multicolumn{3}{|l|}{ Lifestyle } \\
\hline Sedentary & & 79 \\
\hline Active & & 21 \\
\hline Duration of rheumatoid arthritis & $21-0$ & $.36 \pm 8.91$ years \\
\hline Duration of urinary tract & & \\
\hline infection symptoms & & $.67-9.14$ days \\
\hline \multicolumn{3}{|c|}{ Table-II: Common symptoms of urinary tract infection. } \\
\hline \multicolumn{2}{|c|}{ Parameter } & Frequency \\
\hline \multicolumn{2}{|l|}{ Lower abdominal pain } & 77 \\
\hline \multicolumn{2}{|l|}{ Persistent urge to urinate } & 71 \\
\hline \multicolumn{2}{|c|}{ Burning sensation when urinating } & 82 \\
\hline \multicolumn{2}{|c|}{ Passing frequent, small amounts of urine } & 59 \\
\hline \multicolumn{2}{|c|}{ Cloudy urine } & 89 \\
\hline \multicolumn{2}{|l|}{ Pink or cola color urine } & 69 \\
\hline \multicolumn{3}{|c|}{ Table-III: Bacteria isolated from urinary samples. } \\
\hline Bacteria & & Frequency \\
\hline P. mirabilis & & 41 \\
\hline Escherichia coli & & 21 \\
\hline Acinobater species & & 14 \\
\hline Klebsiella species & & 10 \\
\hline Streptococcus spp. & & 8 \\
\hline Enterococcus spp. & & 5 \\
\hline Pseudomonas aeruginosa & & 1 \\
\hline
\end{tabular}

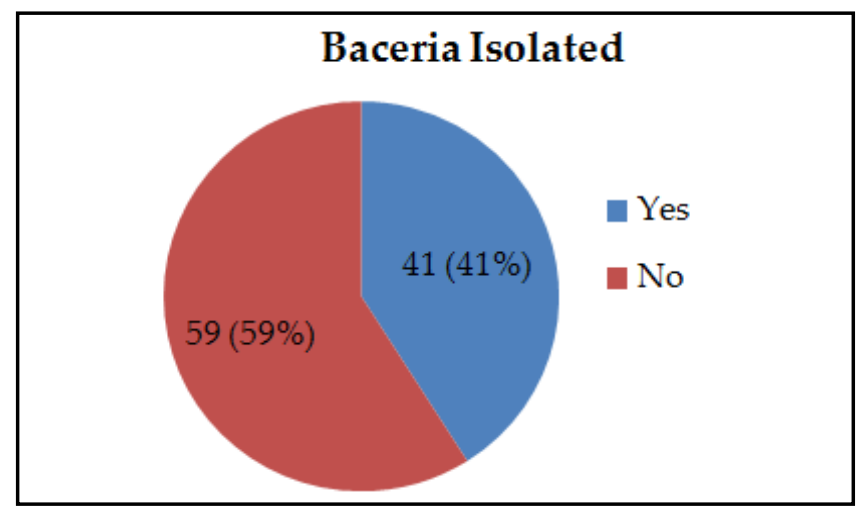

Figure: Distribution of urinary tract infection due to proteus mirabilis. 


\section{DISCUSSION}

$\mathrm{RA}$ is the prolonged inflammatory auto-immune disorder of human beings, which mainly causes pain and swelling in the joints 9 . RA is a complex auto-immune disorder which affects about $1-2 \%$ of general population all over the world. The etiopathogenesis of RA includes the inter-play of several hereditary risk factors and environmental causes ${ }^{10,11}$.

The numerous phenomena are involved in growth of the rheumatoid arthritis, which are not entirely understood and are under debate. It has been suggested that several diverse environmental factors, for example smoking cigarettes, atmospheric and occupational agents can act as triggering stimuli for development of RA in candidates who are inherently exposed to the disease, leading to the synovial hyperplasia and the bone damage. The early stage of RA is related to the variation of native and adaptive immune system, which has consequential production of auto-antibodies, pursuing several molecules together with amended self-epitopes ${ }^{9}$.

A better consideration of how the pathological phenomena initiate the corrosion of RA growth in patients is immediately necessary with the intention of developing the therapies that would successfully manage the patients at every stage of RA progression ${ }^{12}$. Therapies and medications for management of RA are continued to increase promptly ${ }^{13}$.

Urinary tract infection is one of a very common type of infections in local community and vulnerability of uropathogens to commonly applied antibiotics has been decreased during recent years. So it is very important to check to susceptibility patterns of these uropathogens occasionally, with the intention that empirical therapy can be planned by using recent evidence to manage urinary tract infection properly and timely. This may also help to improve the outcome of patients having urinary tract infection, particularly in patients complicated with rheumatoid arthritis. This may also help to minimize the failure of empirical therapies and also help to prevent the misuse of antibiotics ${ }^{14}$.

In a very recently conducted multi-centre trial, which involved nine Spanish hospitals, 784 females with uncomplicated cystitis were examined for the occurrence of isolated uro-pathogens and their susceptibility to several antibiotics. Out of 650 isolated pathogens, the most frequent bacterial agents detected was Escherichia coli (79.2\%), which was followed by staphylococcus saprophyticus (4.4\%), P.mirabilis (4.3\%), enterococcus faecalis $(3.3 \%)$, and klebsiella pneumoniae $(2.3 \%)^{15}$.
In our study, the mean age of patients was $61.22 \pm$ 10.18 years. There were 67 (67\%) females and 33 (33\%) males. About 79 (79\%) patients had sedentary lifestyle due to RA and feeling pain during some work. The mean duration of RA diagnosis was $21.36 \pm 8.91$ years and patients were already taking treatment for RA. They presented with symptoms of urinary tract infection for about $25.67 \pm 9.14$ days duration.

In our study, we observed the most common symptom of urinary tract infection was cloudy urine 89 $(89 \%)$, followed by burning sensation during urination $(82 \%)$, lower abdominal pain $77 \%$, persistent urge to urinate in $71 \%$, pink or cola color urine in $69 \%$ while 59 had passing frequent urine. On urine culture, there were $41(41 \%)$ patients had positive urine culture with P. mirabilis, which is a high percentage and cannot be ignored.

Senior et al, done one study on 76 patients who had RA. Those patients had history of having high antibody levels in their serum, explicit to P.mirabilis. Infection of P.mirabilis bacteriuria in patients of RA was observed in $52 \%$ cases $^{6}$.

RA is still one of the chronic inflammatory disease with unidentified etiologies. The associated risk factors are: tyrosine phosphatase gene, HLA-DR genes, genes of cytokine coding, peptidylarginine deiminase gene ${ }^{16}$. There are several types of herbal treatments which can be used in local Australian traditional medicinal systems to manage RA and inflammation ${ }^{17}$.

RA is a systemic and arthritic autoimmune disease affecting millions of people throughout the world. During the last 4 decades extensive data indicate that subclinical urinary tract infection by P.mirabilis has a role in the aetiopathogenesis of RA based on cross-reactivity or molecular mimicry between Proteus haemolysin and RA-associated HLA-DRB1 alleles as well as between Proteus urease and type XI collagen ${ }^{18}$. In another study, conducted by Cock et al found that with herbal preparations are applied in customary African medicines to manage RA and inflammation, $85.3 \%$ extracts avert the growth of P.mirabilis and $67.7 \%$ of them tested rectified the growth of P.vulgaris ${ }^{19}$.

Raised levels of antibodies against P.mirabilis have been discovered in patients with RA in many inhabitants of fourteen countries ${ }^{20}$. Moreover, in patients with rheumatoid arthritis, there were significant raised concentration of antibodies to P.mirabilis but not against $>20$ other microbes, including E. coli ${ }^{21}$. These antibodies will attach to the joint tissue and will be cytopathic to the joint tissues, which transport the proteus 
cross-reactive antigens and this immune system in reaction will release the more self-tissue antigens with following secretion of further auto-antibodies, proliferation of pathological progression and development of classical rheumatoid arthritis, like Streptococcus causes rheumatic fever \& valvular complications in cardiac muscles 22,23 .

Raised concentrations of anti-bodies to the P.mirabilis have been identified in the patients having RA in different populations from fourteen different countries including United Kingdom, United states of America, England \& Netherlands ${ }^{20}$. The specificity of the proteus anti-bodies in the patients of RA has been reported in several studies. Deighton et al, observed that antibodies against P.mirabilis, other than the pathogens involved in urinary sample were significantly high in RA patients ${ }^{24}$. Furthermore, in one review analysis, it has been reported that in patients of rheumatoid arthritis, there were significant high concentrations of anti-bodies against P.mirabilis, but those were not against $>20$ other entero-bacterial or uro-pathogenic microorganisms, like E. coli21.

As a consequence of molecular mimicry or resemblance in proteus and self-antigens, patients having infection due to proteus species would yield not only the antibodies against this microorganism, but also contrary to the self-tissue molecules transporting the crossreactive antigens ${ }^{11}$. These antibodies would bind to and be cytopathic to the tissue of the joint which transport the proteus cross-reactive antigens. Also, this protected response may lead to the secretion of more selftissue antigens with the consequential production of further auto-antibodies, proliferation of the pathological procedure and the growth of the classical RA. Similarly, Streptococcus causing the rheumatic fever and valvular lesions inside the cardiac muscles ${ }^{22,23}$.

Urinary tract infection is deliberated to be the most common type of infection in human beings and disturbing either; upper kidney or bladder in many ways. RA is most possibly initiated or caused infection in upper urinary tract cause of bacteria known as proteus bacteria ${ }^{20}$.

In another study, Chandrashekar et al, it was observed that whether medical therapy can raise the concentration of anti-Proteus antibodies in patients who had rheumatoid arthritis. The blood samples of 32 patients who had RA were obtained after one year, who were recruited in one of the previous studies and were continued to contribute in the follow-up study. All the patients were prescribed to take methotrexate along with hydroxychloroquine with sufficient dose of non-steroidal anti-inflammatory drug. After one year, 11 cases were in clinical lessening, while remaining 21 patients were still in state of vigorous disease. Antiproteus anti-bodies titres were considerably high in cases of RA after one year of continuous treatment. However, this rise was not significantly different in patients who had clinical remission and those who were in state of vigorous disease ${ }^{25}$.

\section{CONCLUSION}

The high frequency of P.mirabilis infection has been detected in this study and the frequency was of females with complaint of urinary tract infection was high. So there is a need to screen the RA patients of urinary tract infection regularly and suggest them some preventive measures to avoid urinary tract infection in order to avoid P.mirabilis infection.

\section{CONFLICT OF INTEREST}

This study has no conflict of interest to be declared by any author.

\section{REFERENCES}

1. Chen J, Wright K, Davis JM, Jeraldo P, Marietta EV, Murray J, et al. An expansion of rare lineage intestinal microbes characterizes rheumatoid arthritis. Genome Med 2016; 8(1): 43-46.

2. Brusca SB, Abramson SB, Scher JU. Microbiome and mucosal inflammation as extra-articular triggers for rheumatoid arthritis and autoimmunity. Curr Opin Rheumatol 2014; 26(1): 101-7.

3. Zhang X, Zhang D, Jia H, Feng Q, Wang D, Liang D, et al. The oral and gut microbiomes are perturbed in rheumatoid arthritis \& partly normalized after treatment. Nat Med 2015; 21(8):895-5.

4. Roszyk E, Puszczewicz M. Role of human microbiome and selected bacterial infections in the pathogenesis of rheumatoid arthritis. Reumatol 2017; 55(5): 242-50.

5. Armbruster CE, Mobley HL. Merging mythology and morphology: the multifaceted lifestyle of Proteus mirabilis. Nat Rev Microbiol 2012; 10(11): 743-54.

6. Senior BW, Anderson GA, Morley KD, Kerr MA. Evidence that patients with rheumatoid arthritis have asymptomatic 'non-significant' proteus mirabilis bacteriuria more frequently than healthy controls. J Infect 1999; 38(2): 99-6.

7. Cock I. The early stages of rheumatoid arthritis: New targets for the development of combinational drug therapies. OA Arthritis 2014; 2(1): 5-8.

8. Christopoulos G, Christopoulou V, Routsias J, Babionitakis A, Antoniadis C, Vaiopoulos G. Greek rheumatoid arthritis patients have elevated levels of antibodies against antigens from Proteus mirabilis. Clin Rheumatol 2017; 36(3): 527-35.

9. Calabresi E, Petrelli F, Bonifacio AF, Puxeddu I, Alunno A. One year in review 2018: pathogenesis of rheumatoid arthritis. Clin Exp Rheumatol 2018; 36(2): 175-84.

10. Li S, Yu Y, Yue Y, Zhang Z, Su K. Microbial infection and rheumatoid arthritis. J Clin Cell Immunol 2013; 4(6): 174-78.

11. Ebringer A, Rashid T. Rheumatoid arthritis is caused by a proteus urinary tract infection. Apmis 2014; 122(5): 363-8.

12. Guo Q, Wang Y, Xu D, Nossent J, Pavlos NJ, Xu J. Rheumatoid arthritis: pathological mechanisms and modern pharmacologic therapies. Bone Res 2018; 6(15): 1-14. 
13. Mahajan TD, Mikuls TR. Recent advances in the treatment of rheumatoid arthritis. Curr Opin Rheumatol 2018; 30(3): 231-7.

14. Patel H, Soni S, Bhagyalaxmi A. Causative agents of urinary tract infections and their antimicrobial susceptibility patterns at a referral center in Western India: An audit to help clinicians prevent antibiotic misuse. J Fam Med Prim Care 2019; 8(1): 154-9.

15. Palou J, Pigrau C, Molina I, Ledesma JM, Angulo J. Etiology and sensitivity of uropathogens identified in uncomplicated lower urinary tract infections in women (ARESC Study): implications on empiric therapy. Med Clin 2011; 136(1): 1-7.

16. Kotulska A, Kucharz EJ, Wiland P, Olesińska M, Felis-Giemza A, Kopeć-Mędrek M, et al. Satisfaction and discontent of Polish patients with biological therapy of rheumatic diseases: results of a multi-center questionnaire study. Reumatol 2018; 56(3): 140-44.

17. Cock IE, Winnett V, Sirdaarta J, Matthews B. The potential of selected Australian medicinal plants with anti-proteus activity for the treatment and prevention of rheumatoid arthritis. Pharmacog Magaz 2015; 11(Suppl-1): S190-8.

18. Wilson C, Rashid T. Worldwide links between proteus mirabilis and rheumatoid arthritis. J Arthritis 2015; 4(1): 142-45.
19. Cock IE, van Vuuren SF. Anti-proteus activity of some South African medicinal plants: their potential for the prevention of rheumatoid arthritis. Inflammopharmacol 2014; 22(1): 23-36.

20. Ebringer A, Rashid T, Wilson C. Rheumatoid arthritis, Proteus, anti-CCP antibodies and Karl Popper. Autoimmun Rev 2010; 9(4): 216-23.

21. Rashid T, Ebringer A. Rheumatoid arthritis is linked to proteusthe evidence. Clin Rheumatol 2007; 26(7): 1036-43.

22. Wilson C, Rashid T, Tiwana H, Beyan H, Hughes L, Bansal S, et al. Cytotoxicity responses to peptide antigens in rheumatoid arthritis and ankylosing spondylitis. J Rheumatol 2003; 30(5): 972-8.

23. Guilherme L, Köhler K. Rheumatic heart disease: mediation by complex immune events. Adv Clin Chem 2011; 53(2): 31-50.

24. Deighton C, Gray J, Bint A, Walker D. Specificity of the proteus antibody response in rheumatoid arthritis. Ann Rheumat Dis 1992; 51(11): 1206-7.

25. Chandrashekara S, Patil R, Vadiraja HS, Shobha A. The incidence of proteus mirabilis infection increases in patients on treatment but does not trigger disease activity. Clin Rheumatol 2006; 25(4): 520-3. 